\title{
La malattia di Anderson-Fabry. Conclusioni
}

\author{
Giovanni Duro' ${ }^{1}$, Marco Lombardi \\ ${ }^{1}$ Centro di Ricerca e Diagnosi Malattie da Accumulo Lisosomiale, Istituto di Biomedicina e Immunologia Molecolare "A. Monroy" (IBIM), \\ Consiglio Nazionale delle Ricerche (CNR), Palermo \\ ${ }^{2}$ Nefrologia e Dialisi, Ospedale del Mugello, A.S. Toscana Centro, Borgo San Lorenzo (FI)
}

\begin{abstract}
Anderson-Fabry disease. Conclusion
Fabry disease is a hereditary, progressive and multisystemic disorder characterized by variable clinical manifestations and a course that can lead to the death of the patient between the fourth and fifth decades of life if not promptly diagnosed. It is a metabolic, lysosomal storage disorder which is due to the functional deficit of the enzyme alpha-galactosidase $A(\alpha-G A L A)$. The deficit alters the metabolism of some glycosphingolipids, mainly globotriaosylceramide (Gb3) and lyso-Gb3 (the deacetylated form), causing their storage in lysosomes of various cell types, especially those of the vascular endothelium. The knowledge of this disease has improved in recent years and it is increasingly clear that it could be a more complex disorder than previously thought and that also other factors are likely to be involved in the onset of its symptoms.
\end{abstract}

Keywords: $\alpha-G A L A$, Fabry disease, Lyso-Gb3

La Fabry è una malattia ereditaria, progressiva e multisistemica, caratterizzata da manifestazioni cliniche e decorso variabili che possono portare alla morte del paziente entro la quinta decade di vita, se non tempestivamente diagnosticata (1). Si tratta di un disordine metabolico, da accumulo lisosomiale, dovuto al deficit funzionale dell'enzima $\alpha$-galattosidasi A ( $\alpha$-Gal A). Tale deficit determina un'alterazione del metabolismo di alcuni glicosfingolipidi, prevalentemente globotriaosilceramide (Gb3) e lyso-Gb3 (forma deacetilata), che di conseguenza si accumulano nei lisosomi di numerosi tipi cel-

Accepted: June 26, 2017

Published online: July 31, 2017

Indirizzo per la corrispondenza:

Prof. Giovanni Duro

Centro di Ricerca e Diagnosi

Malattie da Accumulo Lisosomiale

Istituto di Biomedicina

e Immunologia Molecolare "A. Monroy"

Consiglio Nazionale delle Ricerche

Via Ugo La Malfa, 153

90146 Palermo

giovanni.duro@ibim.cnr.it

Dr. Marco Lombardi

Nefrologia e Dialisi

Ospedale del Mugello

Viale della Resistenza, 60

50032 Borgo San Lorenzo (FI)

lombardim@tin.it lulari, soprattutto nelle cellule dell'endotelio vascolare (2).

Negli ultimi anni sono aumentate enormemente le conoscenze su questa patologia ed è sempre più chiaro che la Fabry potrebbe essere una patologia più complessa di quanto sia ritenuto e probabilmente, nell'insorgenza della sintomatologia, sono coinvolti anche altri fattori.

L'accumulo di Gb3 e lyso-Gb3, è ancora considerato come la causa principale del fenotipo clinico di Fabry. Tuttavia, i meccanismi esatti che sono coinvolti nello sviluppo della malattia non sono completamente compresi (3). È inoltre compromessa la degradazione di altri glicosfingolipidi, ma il loro contributo allo sviluppo della patologia non è ben noto.

I test biochimici mostrano che i maschi sono caratterizzati da assenza o riduzione significativa dell'attività enzimatica (4) e un marcato aumento di lyso-Gb3 nel plasma e nelle urine (5). L'accumulo di questi glicosfingolipidi complessi, dimostrato da caratteristici inclusioni lisosomiali lamelliformi, può essere trovato in tutti i tessuti e in diversi tipi di cellule, tra cui le cellule vascolari, l'endotelio, i cardiomiociti, i podociti e i neuroni, nei gangli e nel sistema nervoso centrale. II lysoGb3 è stato identificato come un marcatore affidabile di Fabry (5) tuttavia i meccanismi con cui si forma e come esso provoca l'eventuale effetto patologico sono ancora sconosciuti. La complessità della malattia è anche testimoniata dalla mancata corrispondenza, in alcuni casi, tra attività enzimatica e manifestazioni cliniche, in soggetti che presentano mutazioni nel gene GLA. Nella nostra esperienza, basata su più di 15.000 soggetti studiati negli ultimi anni, abbiamo documentato vari casi di familiari dei probandi, di sesso maschile ed età avanzata, che, sebbene presentassero mutazioni in GLA 
e attività enzimatica nulla o fortemente deficitaria, non presentavano manifestazioni cliniche severe (6). Viceversa, nella maggior parte dei probandi da noi studiati, pur presentando manifestazioni cliniche riconducibili alla malattia, non sono state riscontrate mutazioni in GLA responsabili del fenotipo. In entrambi i casi non è chiaro se questi soggetti possono essere considerati affetti dalla malattia di Fabry. In soggetti con sintomi sistemici riconducibili anche alla malattia di Fabry probabilmente dovrebbero essere studiati altri geni, correlati alle manifestazioni sistemiche, in cui eventuali mutazioni potrebbero sommarsi, con effetto sinergico, alle alterazioni nei sistemi implicati nella malattia di Fabry. In questa tipologia di pazienti andrebbero studiati anche i meccanismi implicati nell'autofagia e nel rilascio di esosomi. Secondo la letteratura l'accumulo del substrato specifico, collegato all'enzima mutato, congiuntamente alla de-regolazione di altri meccanismi, provocherebbe il blocco dell'autofagia e conseguentemente il danno cellulare (7). Una interessante ipotesi prevede che nella malattia di Fabry per bloccare l'autofagia possano essere sufficienti livelli relativamente bassi di lyso-Gb3. Questo spiegherebbe la sintomatologia anche nei soggetti con mutazioni dubbie e con livelli di lyso-Gb3 considerati borderline/non patologici. Inoltre noi riteniamo che, come evidenziato in altri sistemi (8), il blocco dell'autofagia possa essere correlato con l'aumento del rilascio di esosomi (o altre vescicole), arricchiti in lyso-Gb3, che rilasciati sistemicamente e interagendo con l'endotelio vascolare, potrebbero essere una concausa del danno evidenziato nei pazienti.

La disponibilità di una terapia enzimatica sostitutiva ricombinante (ERT) ha avuto un importante impatto sulla gestione clinica dei pazienti affetti, modificandone la prognosi e la qualità di vita (9). I dati disponibili in letteratura concordano sull'importanza di un intervento terapeutico specifico il più precoce possibile, prima che il coinvolgimento d'organo diventi irreversibile. Gb3 e lyso-Gb3 sono utilizzati nel followup dei pazienti, in particolare per valutare l'efficacia del trattamento terapeutico. Con la terapia enzimatica sostitutiva (ERT) si osserva una riduzione di Gb3 e lyso-Gb3, ma in qualche caso, dopo qualche trattamento il sistema immunitario reagisce diminuendo l'efficacia della terapia. In conclusione questa patologia dovrebbe essere sempre presa in considera- zione quando giungono all'osservazione pazienti con decorsi clinici atipici, diagnosi incerte, o quadri con coinvolgimento sistemico non chiari. In soggetti adulti in cui vengono rilevati ictus criptogenetico, cardiomiopatia ipertrofica o danno renale può essere sospettata la forma classica o possibili varianti atipiche della malattia. Anche lo studio della storia clinica familiare può indirizzare il medico, tenendo conto della trasmissione della patologia legata al cromosoma $X$.

Una diagnosi precisa e tempestiva è essenziale per avviare precocemente i pazienti alla terapia enzimatica sostitutiva, che è in grado di arrestare o rallentare la progressione della malattia, migliorandone così la qualità della vita.

\section{Disclosures}

Financial support: No financial support was received for this submission.

Conflict of interest: The authors have no conflict of interest.

\section{Bibliografia}

1. Germain DP. Fabry disease. Orphanet J Rare Dis. 2010;5:30.

2. Desnick RJ, loannou YA, Eng CM. Alpha-galactosidase A deficiency: Fabry disease. In: Scriver CR, Beaudet AL, Sly WS, Valle $\mathrm{D}$, eds. The metabolic and molecular basis of inherited disease. $8^{\text {th }}$ ed. New York: McGraw-Hill;2001:3733-74.

3. Spada M, Kasper D, Pagliardini V, Biamino E, Giachero S, Porta F. Metabolic progression to clinical phenotype in classic Fabry disease. Ital J Pediatr. 2017:43;(1):1.

4. Zizzo C, Monte I, Pisani A, et al. Molecular and clinical studies in five index cases with novel mutations in the GLA gene. Gene. 2016;578(1):100-4.

5. Krämer J, Weidemann F. Biomarkers for diagnosing and staging of Fabry disease. Curr Med Chem. 2017 Jun 16.

6. Cammarata G, Fatuzzo P, Rodolico MS, et al. High variability of Fabry disease manifestations in an extended Italian family. Biomed Res Int. 2015;2015:504784.

7. Micsenyi MC, Walkley SU. The Lysosomal System. Lysosomal Storage Disorders. John Wiley \& Sons, Ltd; 2012:1-12.

8. Colomba P, Cammarata G, Zizzo C, et al. Anderson-Fabry: una malattia rara? G Tec Nefrol Dial 2015; 27(2): 73-7.

9. El Dib RP, Nasciento P, Pastores GM. Enzyme replacement therapy for Anderson-Fabry disease. Cochrane Database Syst Rev. 2013;2 CD006663. 